\title{
BISCHOF THIDDAG UND DAS BISCHOFSIDEAL DER REICHSKIRCHE AM ANFANG DES 11. JAHRHUNDERTS
}

\author{
DRAHOMÍR SUCHÁNEK \\ Philosophische Fakultät, Karls-Universität, Prag \\ E-mail: drahomir.suchanek@ff.cuni.cz
}

\begin{abstract}
Bishop Thiddag and the Ideal of Bishop in the Imperial Church in the Beginning of the 11 th Century

The paper thinks through the development of the ideal of the bishop in the 10th and 11 th century. It points out the transformation of the former picture of an ascetic prelate to the self-confident administrator of the entrusted diocese, as it is given by the sources presenting the life and career of the bishop of Prague - Thiddag. While the sources of the 10th century give us the monkish ideal of the bishop's work, a rather traditional model of a saintly bishop, living a humble life, devoted to the asceticism and fulfilling his duties diligently, but with a certain self-denial; the ideal of the 11th century shifts to the selfconfident bishops, managing their administrative duties and even the mundane tasks without any trouble, not desiring to run away to the monastic solitude or regretting their work for the Empire. Their relationship towards their diocese is confirmed by the efforts to enlarge its properties and rights, fulfilling thusly the work begun by their predecessors. The comparison focused on the development of the ideal of the bishop allows us to think deeply about the circumstances and causes bringing the changes of the priorities of the bishops in this very important period leading to the Gregorian reform.
\end{abstract}

Keywords: bishop; Imperial Church; Holy Roman Empire; 11th century

Über den Prager Bischof Thiddag wissen wir nicht allzu viel. Seine Lebensgeschichte fesselte keinen seiner Zeitgenossen so weit, dass er ihm eine ausführliche Abhandlung gewidmet hätte, und er erfreute sich auch nicht des Rufs eines Heiligen oder außerordentlich fähigen Verwalters seines Bistums, dass für ihn ein hagiografischer Text oder eine bischöfliche Lebensbeschreibung (Vita) bestimmt worden wäre. Unsere Hauptquellen für Informationen - Thiddags bischöflicher Mitbruder Thietmar von Merseburg ${ }^{1}$ und der Dekan des Prager Sankt-Veit-Domkapitels Cosmas - schilderten seine Lebensschicksale nur in einigen kurzen Sätzen ${ }^{2}$. Nur schwer setzen wir daher das Bild des dritten Prager Bischofs zusammen und wir können nur raten, ob sein Wirken von den Zeitgenossen positiv oder eher kritisch bewertet wurde. Einerseits ist Thiddag für uns der stille Zeuge einer sehr schweren Zeit in der böhmischen Geschichte zur Wende des ersten

1 Thietmari Merseburgensis episcopi Chronicon (MGH Scriptores rerum Germanicarum. Nova series. Bd. 9), hg. von Robert HOLTZMANN. Berlin 1935, S. 468 ff. (VII 56).

2 Die Chronik der Böhmen des Cosmas von Prag (Cosmae Pragensis Chronica Boemorum) (MGH Scriptores rerum Germanicarum. Nova series. Bd. 2), hg. von Berthold BRETZHOLZ. Berlin 1923, S. 56 (I 31) und S. 72 (I 39). 
und zweiten Jahrtausends, als die Regierung der Přemyslidenfürsten eine Krise erlebte und in der Gesellschaft innere Wirren herrschten ${ }^{3}$. Thietmar verdeckt Bischof Thiddags kompliziertes Wirken in keiner Weise und verweist darauf, dass Thiddag mehrmals den Bischofsstuhl verlassen musste. Er wusste zu schätzen, dass der Prager Bischof in seinem Amt zu verharren vermochte, und sieht vielleicht deshalb mit gewisser Benevolenz über Thiddags Vorliebe für den übermäßigen Alkoholgenuss hinweg. Er glaubte, dass Thiddag an dieser „schlechten Eigenschaft“ keine Schuld trug, und hoffte, dass dieser sich vor seinem Tod „mit guten Arzneien, die seine Seele heilten“ vorzubereiten vermochte ${ }^{4}$.

Das Fehlen detaillierter Angaben zum Leben von Bischof Thiddag, ebenso wie einer tiefen gehenden Abhandlung über seine moralischen Qualitäten, macht eine etwas verborgene Gestalt inmitten des Rests des Reichsepiskopats aus ihm. Bei näherer Betrachtung zeigen sich uns jedoch einige wichtige Charakteristik seines persönlichen und öffentlichen Lebens, die es uns ermöglichen, diesen Prager Bischof in den weiteren Kontext der Sichtweise des Bischofsamtes zur Wende des ersten und zweiten Millenniums einzuordnen. Thietmar erwähnt, dass Thiddag seine Bildung im Kloster Corvey erlangte, so dass er wahrscheinlich aus einer adligen Familie stammte, und seine Beförderung auf den Bischofsstuhl hatte er einerseits seinen medizinischen Fähigkeiten und andererseits der Gunst des regierenden Fürsten Boleslav II. zu danken, dessen Krankheit er zu lindern vermochte. Der Chronist Cosmas betont, dass ihm vor allem Kaiser Otto III. zum Bischofsamt verholfen haben soll und dass seine Kenntnis der slawischen Sprache eine wichtige Rolle spielte. In gewissem Sinn handelt es sich um die typische Kirchenkarriere eines Reichsbischofs, wie sie uns über das ganze 10. Jahrhundert mit Dutzenden bischöflicher Lebensbeschreibungen und Hagiografien belegt wird ${ }^{5}$. Adlige Abstammung, Klosterbildung, außerordentliche Frömmigkeit, eine bestimmte spezifische Fähigkeit (bei

3 Zur Krise des frühen Premyslidenstaates vgl. z. B. Barbara KRZEMIEŃSKA, Krize českého státu na přelomu století, in: $\check{C}$ s $\breve{C} H 18$ (1970), S. 497-532; Marzena MATLA-KOZŁOWSKA, Pierwsi Przemyślidzi i ich państwo (od X do połowy XI wieku): ekspansja terytorialna i jej polityczne uwarunkowania. Poznań 2008, S. 329-399, bzw. David KALHOUS, Boleslav III.: kníže na konci časů?, in: Tomáš Borovský - Libor Jan - Martin Wihoda (Hgg.). Ad Vitam et Honorem: profesoru Jaroslavu Mezníkovi prátelé a žáci k pětasedmdesátým narozeninám. Brno 2003, S. 221-229.

4 „[...] de qua post mortem Bolizlavi senioris ab equivoco eius et filio sepe expulsus, toties a marchione Ekkihardo reducitur, et magnas patitur iniurias. Hic hospites, ut sanctus iubet Gregorius, non solum ad se invitavit, sed etiam traxit, hoc maximum habens vitium, quod ob morbum sibi innocentem bibebat supra modum. Paraliticus enim erat, manuum tremore assiduo sine asstantium auxilio presbiterorum missam canere non potuit; sicque usque ad finem languescens, bonis, ut spero, animam curabat medicaminibus." Thietmari Chronicon, S. 469 f. (VII 56).

5 Eine repräsentative Auswahl der bedeutendsten bischöflichen Lebensläufe stellte Hatto Kallfelz in einer lateinisch-deutschen Edition zusammen (Hatto KALLFELZ, Lebensbeschreibungen einiger Bischöfe des 10.-12. Jahrhunderts. Darmstadt 1973), andere Quelleditionen in: Monumenta Germaniae Historica, vor allem Georg Heinrich PERTZ u. a. (Hg.), Scriptores (in Folio). Bd. 4: Annales, chronica et historiae aevi Carolini et Saxonici. Hannover 1841 und DERS. (Hg.), Scriptores (in Folio). Bd. 11: Historiae aevi Salici. Hannover 1854. An Fachliteratur kann zu dem Thema die erschöpfende Analyse von bischöflichen Viten im 10. und 11. Jahrhundert von Stephanie Haarländer empfohlen werden (Stephanie HAARLÄNDER, Vitae episcoporum: eine Quellengattung zwischen Hagiographie und Historiographie, untersucht an Lebensbeschreibungen von Bischöfen des Regnum Teutonicum im Zeitalter der Ottonen und Salier. Stuttgart 2000), bzw. Michel SOT, Gesta episcoporum, gesta abbatum. Turnhout 1981; Reinhold KAISER, Die Gesta episcoporum als Genus der Geschichtsschreibung, in: Anton Scharer - Georg Scheibelreiter (Hgg.), Historiographie im frühen Mittelalter. Wien 1994, S. 459-480 usw. 
Thiddag die Medizin bzw. Kenntnis der Sprache) sowie die Gunst des Herrschers gehörten zu den unerlässlichen Voraussetzungen des Karriereaufstiegs ${ }^{6}$. Die Zeit von Thiddags Episkopat kann jedoch als eine Periode bezeichnet werden, in der es zu einer allmählichen Veränderung der gewohnten Gepflogenheiten kommt. Der angestrebte Weg zur Erlangung der Bischofswürde, die Prioritäten des Bischofsamts und die Äußerungen der persönlichen Frömmigkeit der einzelnen Bischöfe stellen sich in den Quellen abweichend von denen zum Beispiel Mitte des 10. Jahrhunderts dar, was eine Verschiebung im Verständnis des sog. Bischofsideals andeutet. Von diesem Gesichtspunkt stellt für uns Bischof Thiddag eine einzigartige Gelegenheit dar, tiefer über die Umstände und Ursachen des Wandels der Bischofsprioritäten in einer wichtigen Epoche nachzudenken, die in den gregorianischen Reformen gipfelt.

\section{Das Bischofsideal im Licht der Quellen}

Das Grundproblem bei der Suche nach dem zeitgenössischen Ideal eines Bischofs ist im frühen Mittelalter wie im Hochmittelalter mit den Quellen und deren Interpretation verbunden. Bis auf ausgesprochene Ausnahmen entstanden keine Mustertexte, die das erstrebte Bild eines Bischofs bzw. seines persönlichen oder öffentlichen Lebens präsentieren würden. Die Verfasser der mittelalterlichen Texte konnten selbstverständlich bei ihren Präsentationen auf einige Schriften zurückgreifen, die in den vorangegangenen Jahrzehnten oder Jahrhunderten die Stellung fast verbindlicher „Handbücher“ zur Beurteilung der Qualitäten von Inhabern des Bischofsamts erlangt hatten. Jedoch handelt es sich nicht um eine Art Aretalogiekataloge, also um die formelle Aufzählung erstrebenswerter Tugenden, geeigneter Charakterzüge und praktischer Tätigkeiten, die mit dem Bischofsamt vereinbar sind. Außerordentliche Aufmerksamkeit erfreute sich insbesondere die Regula pastoralis von Papst Gregor I., aus der gern zitiert wurde und auf die sich die Verfasser der bischöflichen Viten häufig als auf das praktische und theoretische Vorbild für die Verwaltung eines Bistums beriefen ${ }^{7}$. Die gebildeteren Kleriker vermochten Inspiration auch in den antiken moralischen Texten zu schöpfen, insbesondere wenn diese anschließend von christlichen Autoritäten rezipiert wurden (z. B. in der Schrift De officiis des Ambrosius von Mailand, der an das gleichnamige Werk Ciceros anknüpfte) ${ }^{8}$. Andere theoretische Werke nach dem Muster der Fürstenspiegel haben wir jedoch für Bischöfe nicht, wenn wir die Praeloquia

6 Eine Analyse der Bischofskarrieren in der ottonisch-salischen Zeit bringt zahlreiche hochwertige Arbeiten, verwiesen sei vor allem auf die bereits klassischen Werke von Albrecht FINCK VON FINCKENSTEIN, Bischof und Reich: Untersuchungen zum Integrationsprozess des ottonisch-frühsalischen Reiches (919-1056). Sigmaringen 1989; Herbert ZIELINSKI, Der Reichsepiskopat in spätottonischer und salischer Zeit (1002-1135). Wiesbaden 1984 oder Odilo ENGELS, Der Reichsbischof in ottonischer und frühsalischer Zeit, in: Irena Crusius (Hg.), Beiträge zu Geschichte und Struktur der mittelalterlichen Germania Sacra. Göttingen 1989, S. 135-175 usw.

7 Siehe Gregor der Große, Regula pastoralis: wie der Seelsorger, der ein untadeliges Leben führt, die ihm anvertrauten Gläubigen belehren und anleiten soll, hg. u. übers. von Georg KUBIS. Graz 1986. Vgl. auch Silke FLORYSZCZAK, Die „Regula pastoralis“ Gregors des Großen: Studien zu Text, kirchenpolitischer Bedeutung und Rezeption in der Karolingerzeit. Tübingen 2005 und Helmut LIPPELT, Thietmar von Merseburg: Reichsbischof und Chronist. Köln/Wien 1973, S. 29 f.

8 Mehr dazu z. B. Maria BECKER, Die Kardinaltugenden bei Cicero und Ambrosius: De officiis. Basel 1994. 
von Rather von Verona außer Acht lassen, die eher eine Anleitung zur Führung moralisch angebrachter Polemiken sind? .

Das Fehlen relevanter thematischer Quellen zwingt uns daher, das Bischofsideal aus jenen Quellentypen zu rekonstruieren, die in ihrer Aussage ein anderes Ziel und eine andere Bedeutung ebenso wie eine andere Leserzielgruppe haben können (und zumeist auch haben). An erster Stelle sind dies die bischöflichen Lebensbeschreibungen (vitae), die uns trotz des unbestreitbaren Bemühens, ihre bischöflichen „Helden“ zu idealisieren, eine umfangreiche und gut ausgearbeitete Präsentation des Denkens und Handels während seines Episkopats bieten. Außer ihnen kann eine Reihe von weiteren Texten genutzt werden, bei denen der Blick auf den Bischof entweder fragmentarisch (bischöfliche Gesta, Chroniken, Briefe), oder im Bemühen um seine Lobpreisung stärker verzerrt ist Wundersammlungen (miracula), Lobreden (laudatia) usw. Bereits zu Beginn unserer Analyse ist also auf die methodische Gefahr hinzuweisen, wenn wir uns bemühen sollten, aus diesen vielschichtigen Texten ein Bischofsideal zu modellieren und ihm vielleicht sogar eine erstrebte Verbindlichkeit verleihen wollten; es lässt sich nie ausschließen, dass es sich um ein reines Konstrukt der Geschichtswissenschaft handelt.

Wenn wir die einzelnen Bischofslebensläufe lesen, vor allem die vitae, stellen uns die Autoren zumeist die betreffende Persönlichkeit in idealisierter Form vor. Bei einem Vergleich auch nur weniger Texte aus dem betreffenden Zeitraum ist nicht zu übersehen, dass ihre Verfasser eine sehr ähnliche Schilderungsstruktur des Lebens der Bischöfe von der Geburt bis zum Tod beibehielten, ähnliche sprachliche und literarische Wendungen sowie Bezüge auf biblische Vorbilder verwendeten und vergleichbare moralische Eigenschaften hervorhoben ${ }^{10}$. Die historisch-literarische Forschung hat schon seit langem bewiesen, dass die mittelalterlichen Quellen von hagiographischem Charakter etliches aus bewährten literarischen, stilistischen und inhaltlichen Vorbildern und Topoi entnahmen, die sie nachahmten und weiter entwickelten, um so die Anforderungen ihrer Lesergemeinde zufrieden zu stellen ${ }^{11}$. Vorbilder wurden hauptsächlich in Texten über außerordentliche Persönlichkeiten gesehen, die in ihrem Leben das asketische Ideal mit dem Bischofsdienst zu vereinen verstanden. Eine zentrale Rolle unter ihnen bewahrte sich der Bischof und asketischer Mönch Martin von Tours, bzw. einige literarische Werke, die dieses Ideal formten (Vita sancti Martini, Dialogi, sowie drei Briefe über den Tod des hl. Martin von Sulpicius Severus $)^{12}$.

9 Zu der Person und dem Werk des Rathers von Verona vgl. Benedetta VALTORTA, Clavis Scriptorum Latinorum Medii Aevi: Auctores Italiae (700-1000). Firenze 2006, S. 240-266, bzw. Bele FREUDENBERG, Emotionen in einem Werk des 10. Jahrhunderts: die Praeloquia Rathers von Verona, in: Jürgen Sarnowsky (Hg.), Bilder - Wahrnehmungen - Vorstellungen: neue Forschungen zur Historiographie des hohen und späten Mittelalters. Göttingen 2007, S. 17-32. Siehe auch HAARLÄNDER, Vitae episcoporum, S. $226 \mathrm{f}$.

10 Detailliert beschäftigt sich mit den biblischen Vorbildern und moralischen Tugenden Stephanie Haarländer in ihrer Arbeit: HAARLÄNDER, Vitae episcoporum, S. 231-253.

${ }_{11}$ Zur Topik allgemein siehe vor allem Lothar BORNSCHEUER, Topik: zur Struktur der gesellschaftlichen Einbildungskraft. Frankfurt am M. 1976, mit Schwerpunkt auf den Anfang des Hochmittelalters vgl. Wolfgang HUG, Elemente der Biographie im Hochmittelalter: Untersuchung zu Darstellungsform und Geschichtsbild der Viten vom Ausgang der Ottonen bis in die Anfänge der Stauferzeit. München 1957.

12 Zusammenfassend zu den literarischen Vorbildern im frühen Mittelalter und zu Beginn des Hochmittelalters siehe Dieter von der NAHMER, Die lateinische Heiligenvita: eine Einführung in die lateinische Hagiographie. Darmstadt 2005, vor allem S. 46-169. Eine Analyse der Sankt-Martins-Quellen bringt 
Die bischöflichen Viten und ihnen ähnliche Texte nur als literarische Fiktion mit vordergründigen hagiographischen Zielen aufzufassen, wäre aber zu eng gesehen. Im Prinzip unterscheiden wir zwei Typen an Lebensbeschreibungen. Einerseits moralistische und erzieherische Biografien mit deutlicher Überlegenheit der ethischen Vorbilder der geschilderten Personen. Die Autoren verwenden hier eine Vielzahl an biblischen Verweisen, vergleichen die Bischöfe mit bedeutenden Persönlichkeiten der christlichen Vergangenheit und präsentieren ihre Lebensbahn als Erfüllung eines von Gott vorbestimmten Plans. Der zweite Typ von Lebensbeschreibungen weicht hiervon etwas ab. Er enthält eine unübersehbare Fülle an konkreten biografischen Elementen aus dem Leben des Bischofs, arbeitet stark mit historischen Ereignissen und Fakten und beschönigt bei Notwendigkeit nicht einen Charakterzug oder eine Tat, die der präsentierten Person in den Augen der Leser schädigen könnte. Eine strikte Trennung beider Biografietypen ist jedoch nicht möglich und man sollte eher von einer Mischform sprechen, in der jeweils eine der angedeuteten Tendenzen überwiegt ${ }^{13}$. Mit einem gewissen Maß an Verallgemeinerung ist zu konstatieren, dass der Entstehungszeitrahmen des betreffenden Textes eine wichtige Rolle spielt. Wenn immer noch Zeitgenossen der vorgestellten Person leben und das kollektive Gedächtnis der Lesergemeinde noch lebendig ist, ist auch die Tendenz erkennbar, den Bischof aufgrund historisch glaubwürdiger und konkreter Ereignisse vorzustellen. Wenn jedoch im Gegenteil das Werk in einem längeren Zeithorizont vom Tod der betreffenden Person verfasst wurde, lassen sich das Fehlen genauerer Informationen bzw. die stärkere Betonung einer literarisch ausgearbeiteten und „schöneren“ Textstilisierung erwarten.

Im gewissen Sinn ermöglichen gerade diese zeitlich entfernteren Biografien dem Autor, sich mehr auf die Präsentation des Bischofsideals zu konzentrieren, das auch das eigentliche Gerüst der Geschichte der beschriebenen Person bildet. Der Verfasser widmet einen größeren Raum allgemeineren Beschreibungen und biblischen Verweisen und nutzt eventuelles Konkretes zur Bestätigung des im Voraus festliegenden Bildes. Der Historiker wird daher stets vor der schweren Aufgabe stehen, zu entscheiden, wie er das analysierte Leben des Bischofs und durch dieses das präsentierte Ideal der Ausübung des Bischofsamts sehen soll. Auf einer Seite steht die Tendenz, es als Norm zu verstehen, die die Lebenspraxis und die Realität des betreffenden Bischofs präsentiert, auf der anderen Seite dagegen eine gewisse Skepsis, dass es sich eher um eine literarische Beschreibung dieser Lebensrealität handelt. Alles wird noch erschwert durch einen Aspekt des Studiums dieser Art mittelalterlicher biografischer Texte, und zwar die wichtige Rolle des Verfassers selbst. Stets sind sorgfältig der Hintergrund, die Ziele und Intentionen der Autoren der bischöflichen Lebensbeschreibungen zu untersuchen und mit der konkreten Situation zu verbinden, in der sie ihre Werke schufen. Es gibt nämlich keinen Zweifel daran, dass die meisten vitae sehr originale Werke sind, die sich zwar an die verbindlichen Schreibmodelle zu halten bemühten, zugleich jedoch ihr eigenes, oft sehr konkretes

z. B. Walter BERSCHIN, Biographie und Epochenstil im lateinischen Mittelalter. Bd. 1: Von der „Passio Perpetuae“ zu den „Dialogi“ Gregors des Grossen. Stuttgart 1986, S. 195-211, bzw. Clare STANCLIFFE, St. Martin and his Hagiographer: History and Miracle in Sulpicius Severus. Oxford 1983, S. 363-371.

13 Vgl. Friedrich LOTTER, Methodisches zur Gewinnung historischer Erkenntnisse aus hagiographischen Quellen, in: Historische Zeitschrift 229 (1979), S. 307-313. 
Ziel verfolgten ${ }^{14}$. Sorgfältige Analysen der Viten aus dem 10. und 11. Jahrhundert haben erwiesen, dass jeder der Autoren mit seinem Werk eine klare Absicht verfolgte, und wichtiger als das Leben und die Eigenschaften der Person, über die geschrieben wird, waren nicht selten die Situation, in der sich der Schreibende befand, und die Probleme, die er zu lösen hatte ${ }^{15}$. Andererseits können wir gerade dank dieser Determinationen verfolgen, worauf und weshalb die Verfasser Betonung legten und wie sich ihre Prioritäten mit der Zeit wandelten.

\section{Das sich ändernde Bild des Bischofsideals}

Die meisten Viten, die im 10. Jahrhundert entstanden, stellen das Ideal des bischöflichen Wirkens im Geist des traditionellen Modells des heiligen Bischofs vor, der ein bescheidenes Leben führt, sich der Askese und Entsagung hingibt und seine bischöflichen Pflichten zwar sorgfältig ausübt, jedoch nicht selten mit Selbstüberwindung. Ein wesentlicher Teil von ihnen erwarb seine Bildung und Vorstellungen über das wünschenswerte Ideal des geistlichen Lebens im Milieu der Klöster. Als sie Bischöfe wurden, übertrugen sie diese Ideale auch auf das Bischofsamt - sie achteten an erster Stelle auf ihre Erlösung, entwickelten die äußeren und inneren Formen ihrer Frömmigkeit weiter und führten in diesem Geist auch die ihnen anvertrauten Kleriker ${ }^{16}$. In diesem Modell werden die drei bedeutendsten Gestalten des Reichepiskopats der zweiten Hälfte des 10. Jahrhunderts vorgestellt - Ulrich von Augsburg, Brun von Köln und Wolfgang von Regensburg. Die Verfasser aller drei Lebensläufe beschäftigen sich noch mit dem Ideal hochfähiger Bischöfe, die voll engagiert sind in der Verwaltung ihrer Bistümer und für die Bedürfnisse des Königreichs, sie zweifeln jedoch nicht daran, dass ein Bischof ständig nach der christlichen Heiligkeit im Geiste der Mönchstugenden streben muss. Deshalb half Ulrich von Augsburg unermüdlich dem König, sogar bei dessen Feldzügen, befestigte seine Residenzstadt Augsburg und engagierte sich im Kampf gegen die Ungarn ${ }^{17}$, zugleich sehnte er sich jedoch danach, ins Kloster zurückzugehen und wollte die Verwaltung des Bistums seinem Neffen Adalbero anvertrauen ${ }^{18}$. Ähnlich half Brun von Köln, oft bezeichnet als

14 Zum Thema vor allem Walter BERSCHIN, Biographie und Epochenstil im lateinischen Mittelalter. Bd. 4: Ottonische Biographie, das hohe Mittelalter: 920-1220 n. Chr. Teil 1: 920-1070. Stuttgart 1999, bzw. in einem ersten Überblick mit weiteren Verweisen auf relevante Literatur siehe HAARLÄNDER, Vitae episcoporum, S. 1-15.

15 Vgl. dazu auch die interessante Studie von Stephanie COUÉ, Acht Bischofsviten aus der Salierzeit neu interpretiert, in: Stefan Weinfurter (Hg.), Die Salier und das Reich. Bd. 3: Gesellschaftlicher und ideengeschichtlicher Wandel im Reich der Salier. Sigmaringen 1991, S. 347-414.

16 Die grundlegenden Aspekte des „monastischen“ Ideals des Bischofs zusammenfasst Daniel ALT, Sanctus episcopus: das Bischofsideal von früh-und hochmittelalterlichen Bischofsviten im Spannungsfeld von Anspruch und Wirklichkeit. Herne 2013, S. 150-166.

17 „Hora vero belli episcopus super caballum suum sedens stola indutus, non clippeo, aut lorica, aut galea munitus, iaculis et lapidibus undique circa eum discurrentibus, intactus et inlesus subsistebat. Bello vero finito, regrediens circuivit civitatem, et domos belli in circuitu civitatis congruenter ponere, et in tota nocte eas aedificare, et vallos, quantum tempus suppetebat, renovare praecepit. Ille autem totum spatium noctis in oratione pernoctans..." Gerhard, Vita S. Oudalrici, hg. von KALLFELZ, Lebensbeschreibungen einiger Bischöfe des 10.-12. Jahrhunderts, S. 104, 106 (Kap. 12).

18 „De hac pro certo sedulitate eius servitii cottidiani concessum est eius avunculo episcopo Oudalrico, ut praefatus Adalbero in eius vice itinera hostilia cum milicia episcopali in voluntatem imperatoris perageret et in curte imperatoris eius vice assiduitate servitii moraretur, ea videlicet causa, ut pra- 
Urbild eines neuen Modells des Reichsbischofs, nach allen Kräften seinem Bruder Otto I. bei der Verwaltung des Reichs und auch bei militärischen Aktionen, dem Biograf Ruotgar zufolge lebte er jedoch gleichzeitig als Asket, strebte nach einer Klosterreform und sein Herz war dem Mönchsdasein zugetan ${ }^{19}$.

Ein Kind dieses Bischofsmodells war offenbar auch Thiddag. Sein Mutterkloster im sächsischen Corvey gehörte lange Zeit zu den Prestigeeinrichtungen, die zahlreiche Kandidaten für den Bischofsstuhl heranzogen ${ }^{20}$. In dem Augenblick, als Thiddag sich der Leitung der Prager Diözese annahm, war jedoch eine Veränderung nicht mehr zu übersehen. Aus den Klostereinrichtungen verschob sich schrittweise die Erziehung perspektivreicher Kleriker zu den Bischofskathedralen und zur königlichen Hofkapelle. Zu neuen Zentren wurden etwa Magdeburg, wo auch der bereits erwähnte Thietmar von Merseburg studierte, oder Hildesheim, das seinen Gipfel in den ersten beiden Dekaden des 11. Jahrhunderts unter der Regierung von Kaiser Heinrich II. erlebte. Von hier wählten sich dann die Herrscher geeignete Kandidaten für ihre Hofkapelle aus und bereiteten sie für den königlichen Dienst vor ${ }^{21}$. Die Bischöfe, die hier ihre Bildung erzogen wurden, sahen ihre Berufung im aktiven Dienst für ihre Diözese, hatten keine Probleme mit Aufgaben in Politik und Verwaltung und identifizierten sich voll und ganz mit den Interessen ihres Bistums. Sorgfältig achten sie auf die Entwicklung der Domkapitel, die zu Zentren der Bildung und Kultur werden und Kleriker hauptsächlich auf die Priesterweihe vorbereiten. Dies sind nicht mehr die Pseudo-Mönchsgemeinschaften, wo das Ideal das gemeinsame Leben der Fratres ist und die Aktivitäten der Kanoniker sich auf die Frömmigkeitsbezeigungen beschränken. Der Bischof steht an der Spitze der gesamten Diözese, umgeben von den Kanonikern als seinem Senat und von seinen Helfern, von denen er eine zunehmend aktive Teilnahme erwartet. Dieser Prozess begann sich zu Thiddags

efato praesuli Dei servicio et custodiae gregis commendati et utilitatibus aecclesiae et orationibus et elemonsinis secundum suum desiderium immorari licuisset. Ille vero quantum secularibus curis se absolutiorem esse persensit, tantum se ipsum in Dei voluntate facere nitebatur obligatiorem." Ebd., S. 64 (Kap. 3).

19 Viz Ruotger, Vita S. Brunonis, hg. von KALLFELZ, Lebensbeschreibungen einiger Bischöfe des 10.-12. Jahrhunderts, vor allem Kap. 29-30, wo Brunos Bescheidenheit, seine Verachtung für Reichtum und Luxus sowie eine Mahnung an den eigenen zukünftigen Tod zu finden sind. Niemals sagt er sich jedoch von seinen Pflichten los, er ist sich seiner Verpflichtungen nach 1 Tim 3, 1-7 bewusst: "Causantur forte aliqui divinae dispensationis ignari, quare episcopus rem populi et pericula belli tractaverit, cum animarum tantummodo curam susceperit [...] Honestum enim et utile nostrae reipublicae fuit omne quod fecit. Factis vero suis hunc finem nequaquam constituit, ut nonnisi per hominum ora favorem captando volarent, sed ita vixit, ita opera sua eoram hominibus temperavit, ut pessimis horrori, bonis esset honori. His aperte cunctis innotuit, quod in episcopatu bonum opus desideravit, in quo nec ab invidis et aemulis suis facile potuit reprehendi, nisi hoc ipsum in laude eius magis proficeret, quod talibus displiceret. Hac igitur mirifica operatione detentus pervigil summi patrisfamilias operator et summus praesul, lucernam ardentem, boni videlicet operis exemplar, in manibus ferens, ad ea quae Dei sunt alios volentes duxit, alios nolentes traxit." Ebd., S. 212, 214 (Kap. 23).

20 Zur Bedeutung des Klosters Corvey für die Reichskirchengeschichte siehe den Beitrag von Daniel Ziemann in diesem Buch.

21 Zur Bedeutung der königlichen Hofkapelle für die Formung der Bischofskandidaten siehe Josef FLECKENSTEIN, Die Hofkapelle der deutschen Könige. Bd. 2. Stuttgart 1966. Mit der steigenden Rolle der Bischofsschulen und kanonischen Kollegiate bei der Erziehung der Bischöfe beschäftigt sich z. B. Johannes LAUDAGE, Priesterbild und Reformpapsttum im 11. Jahrhundert. Köln/Wien 1984, vor allem S. 90-115. 
Zeiten erst durchzusetzen, Ende des 11. Jahrhunderts steht jedoch das Selbstbewusstsein der Kanoniker auf der gleichen geistlichen Stufe wie das der Mönche ${ }^{22}$.

Ebenso wächst die auf die liturgische Seite des Bischofsamts gelegte Betonung. Die Verfasser der Bischofsviten sprechen immer häufiger von schönen und feierlichen Messen und von der Beschaffung neuer liturgischer Gegenstände. Dies bedeutet nicht notwendig, dass die Bischöfe früher die Liturgie vernachlässigt hätten; es sei nur an Ulrich von Augsburg erinnert, bei dem dessen Biograf Gerhard betonte, dass er täglich drei Gottesdienste abhielt und auf deren Feierlichkeit achtete ${ }^{23}$. Neu ist eher die Betonung, die von den Verfassern der Viten auf diesen Bereich der bischöflichen Tätigkeit gelegt wird. Der Gottesdienst ist jetzt eine unerlässliche Bedingung für die Ausübung des Bischofsamts. Dagegen verliert sich nach und nach die frühere Faszination für Askese und körperliches Entsagen. Vom Bischof wird immer noch erwartet, dass er persönlich arm und bescheiden ist, doch ändert sich der Grund, weshalb er so lebt. An erster Stelle steht nicht nach den Mönchsvorbildern die Erlösung des Bischofs, sondern die Weiterentwicklung und Hebung der bischöflichen Kirche, für die er sich bemüht, alles zu opfern. Wie der deutsche Historiker Oskar Köhler richtig betont, die Viten der Bischöfe jener Zeit sehen jene humilitas, d.h. Demut und persönliche Bescheidenheit, als notwendige Forderung für die Ausübung des Bischofsamts, gemeinsam mit dem Dienst am König und der geistlichen Freiheit, und stellen sie auf denselben Rang wie die bischöfliche auctoritas, dignitas oder nobilitas ${ }^{24}$. Übt der Bischof Handlungen der Bußfertigkeit aus, wäscht er z. B. Armen die Beine und bedient er am Tisch, so erfüllt er damit eher die feste Erwartung, die mit dem symbolischen Verhalten des Bischofs an der Öffentlichkeit verbunden ist. Es handelt sich an erster Stelle um eine formalisierte Tätigkeit, um das Prestige und die Würde des Bischofsamts ${ }^{25}$. Deshalb konnte sie auch die stolze Ausübung der bischöflichen Würde sein ${ }^{26}$.

Der Chronist Thietmar drückte den sich vertiefenden Abgrund zwischen diesem neuen Verständnis des Bischofsamts und den Gewohnheiten aus der Vergangenheit am Beispiel des Meißener Bischofs Eido aus, der sich durch übertriebene Askese auszeichnete und übrigens zwei Jahre vor Thiddag starb. Thietmar, der dessen Wirken als Bischof zusammenfasste, konstatierte abschließend: „Uns, seinen Zeitgenossen, gefällt seine Lebensweise wegen unserer eigenen Sünden nicht, ebenso wie ihm die unsere nicht

${ }^{22}$ Mit der Wandlung der Sicht des kanonischen Ideals befasst sich vor allem Georg DENZLER, Die Kanonikerbewegung und die gregorianische Reform im 11. Jahrhundert, in: Studi gregoriani per la storia della Libertas Ecclesiae 9 (1972), S. 223-237; Stefan WEINFURTER, Neue Forschung zur den Regularkanonikern im deutschen Reich des 11. und 12. Jahrhunderts, in: Historische Zeitschrift 224 (1977), S. 379-397, bzw. ENGELS, Der Reichsbischof in ottonischer und frühsalischer Zeit, S. $141 \mathrm{ff}$.

23 Vgl. Gerhard. Vita S. Oudalrici, hg. von KALLFELZ, Lebensbeschreibungen einiger Bischöfe des 10.-12. Jahrhunderts, S. 64 (Kap. 3).

24 Oskar KÖHLER, Das Bild des geistlichen Fürsten in den Viten des 10., 11. und 12. Jahrhundert. Berlin 1935, S. 42. Mehr zu diesem Thema vgl. LIPPELT, Thietmar von Merseburg, S. 24.

25 Zum Beispiel Adalbert von Bremen, der sehr hohen Wert auf Pomp und feierliche Ausführung der weltlichen und geistlichen Akte legte, wusch zugleich als Ausdruck seiner Demut jeden Tag in großem Stil mehr als 30 Armen und Wallfahrern die Füße und verband somit anschaulich adligen Stolz mit dem Bewusstsein seines Amtes. Siehe Adam von Bremen, Gesta Hammaburgensis ecclesiae pontificum, hg. von Georg Heinrich PERTZ u. a., in: MGH Scriptores (in Folio). Bd. 7: Chronica et gesta aevi Salici. Hannover 1846, S. 336 (III 2).

26 Die Transformation eines neuen Typs von Bischof präsentiert vor allem ENGELS, Der Reichsbischof in ottonischer und frühsalischer Zeit, S. 139-175, bzw. ALT, Sanctus episcopus, S. 186-197. 


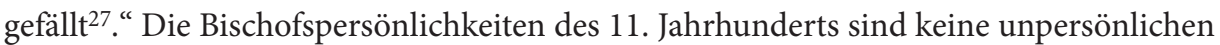
Heiligen, sondern Menschen mit Fehlern und Schwächen, die sie nicht verbergen. Umso mehr benötigen sie aber die Fürbitte der Menschen, und deshalb finden wir auch den bis dahin unbekannten Realismus in den biografischen Viten ${ }^{28}$.

An den soeben erwähnten Beispielen lässt sich dokumentieren, dass sich die Sichtweise des wünschenswerten Bischofsideals schrittweise ändert. Selbstverständlich findet man auch in der ersten Hälfte des 11. Jahrhunderts Viten, die die Betonung auf die monastischen Tugenden des Bischofs legen. Zumeist sind doch ihre Verfasser Mönche und Äbte, wie etwa Richard von Fulda, der die Vita von Erzbischof Willigis von Mainz verfasste. Es entsteht eine Generation selbstbewusster Bischöfe, die ohne Probleme die Verwaltung ihrer Diözesen einschließlich der weltlichen Aufgaben bewältigen, und nicht die Tendenz haben, sich vor ihnen in die Einsamkeit des Klosterlebens zu flüchten oder ihr Tun zu bedauern. Ihre enge Beziehung zur Diözese bestätigen sie mit dem Bemühen, dessen Vermögen und Rechte zu mehren und das zu vollenden, was ihre Vorgänger begonnen haben. Zumeist bedeutet dies, z. B. die Bischofskirche fertig zu bauen oder ein gegründetes Kanonikerstift auszustatten, anderswo wieder die Bestätigung der Rechte des Bistums gegenüber verschiedenen Konkurrenten. Thietmar von Merseburg zeigt ebenfalls eine bestimmte Verantwortung der Bischöfe füreinander. Als er den Tod einzelner Bischöfe erwähnt, schildert er kurz deren Wirken im Amt und denkt über die „Perspektive“ der Erlösung des Verstorbenen nach. Vielleicht ist dies der Ausdruck einer im Entstehen begriffenen bischöflichen Kollegialität, eines tatsächlichen Reichepiskopats als Ganzes.

\section{Das Bischofsideal und die böhmische Kirche}

Es ist natürlich die Frage, inwieweit sich dieser Trend in den böhmischen Verhältnissen niedergeschlagen hat. Thiddag könnten wir wohl noch zur älteren Bischofsgeneration zählen. Auch die beiden Nachfolger Thiddags - Ekkehard und Hizzo - erlangten ihre Bildung und geistliche Formierung noch im Milieu der Klöster und übertrugen ihre monastischen Ideale zweifellos auch auf das Sankt-Veits-Domkapitel, das von beiden als Pröpste geleitet wurde. Cosmas beschrieb ihren Bischofsdienst als konfliktfrei und verantwortungsvoll, erfüllt von Freundlichkeit zu den Armen und Bedürftigen ${ }^{29}$. Die Änderungen setzten erst mit Bischof Šebír (Severus) ein, der seine Karriere in der Nähe des Fürstenhofs gestaltete und nicht so stark mit dem Mönchsmilieu und seinen Idealen verbunden war. Der Gipfel trat dann mit dem Wirken von Bischof Jaromír (Gebhard) ein. Seine Bildung erhielt er an den Domschulen und sein mehrjähriger Aufenthalt im Reich machte ihn zu einem Kirchenprälaten, der großen Wert auf eine selbstbewusste Präsenta-

27 Thietmari Chronicon, S. 844 (VII 25).

28 Ein Beispiel mag die offene Schilderung der Jähzornigkeit des Kölner Erzbischofs Anno bei Lampert von Hersfeld sein (Lampert von Hersfeld, Annales, hg. von Oswald HOLDER-EGGER [MGH Scriptores rerum Germanicarum in usum scholarum separatim editi. Bd. 38]. Hannover 1894, S. 187, a. 1074).

29 Die Chronik der Böhmen, S. 75 f. (I 40-41). 
tion des Bischofsamts legte ${ }^{30}$. Der Chronist Cosmas lobte seine Frömmigkeit, Freigiebigkeit und Demut. Wir hören von seinem alltäglichen Verteilen von 40 Viertellaiben Brot, der Fußwaschung von 12 Wallfahrern, dem Bedienen von Bettlern zu Tisch u. ä., genau nach dem Vorbild, mit dem sich die sonstigen Reichsbischöfe seiner Zeit präsentieren ${ }^{31}$. Gleich nach seiner Ankunft in Prag beginnt er, auch das Kanonikerkapitel zu reformieren. Auch wenn sich den Grund traditionell in den Missständen sieht, die hier geherrscht haben sollen, ist nicht auszuschließen, dass der neue Probst Markus sich primär um die Schaffung einer eigenständigen Institution nach den im Reich bekannten Modellen bemühte ${ }^{32}$. Seine Bereitschaft, den Kanonikern feste Pfründe zuzuweisen und sie mehr in die Verwaltungsaufgaben einzubinden, könnte darauf hindeuten, dass das Imitieren des Vorbilds Mönchsgemeinschaft zu Ende geht und mehr die priesterlichen Aspekte ihres Dienstes betont werden.

Bei Bischof Jaromír (Gebhard) setzt sich endlich die im Reich bereits etablierte Sichtweise des Bischofs in seiner institutionellen Form durch. Jaromír ist sich seiner Pflicht bewusst, das gesamte ihm anvertraute Bistum gut zu verwalten, identifiziert sich voll mit den Interessen und Rechten seines Amtes und kümmert sich um die anvertrauten Kleriker. Nie findet er sich deshalb mit dem Verlust seiner Bischofswürde zugunsten des neuen Bistums Olmütz ab und kämpft bis zum Ende um seine Rechte. Im Gegensatz zu ihm steht der Olmützer Bischof Johann, der noch das ältere Modell symbolisiert. Der Benediktinermönch, der die persönliche Frömmigkeit über die Interessen seines Amtes stellt, was Cosmas mit der Armut illustriert, in der Johann lebte. Jaromír war darüber entsetzt, wie der Olmützer Bischof durch sein Verhalten das Bischofsamt verunglimpfte, und bestrafte ihn sofort. Einfach ausgedrückt - Johann übte sein Amt nicht mit der ihm zustehenden Würde aus, und deshalb war er des Amtes nicht würdig ${ }^{33}$. Dies ist etwas, was der Prager Bischofsklerus in vollem Maß noch nie erlebt hat. Und obwohl jeder Jaromírs Mängel sieht ${ }^{34}$, ist dieses neue Modell eines souveränen und prächtigen Bischofs faszinierend. Der Bischofsklerus identifiziert sich in erheblichem Maß ebenfalls mit diesem Modell. Als die päpstlichen Legaten Jaromír suspendierten und ihm sogar das Recht entzogen, Priesterämter auszuüben, stellte sich der Klerus hinter seinen Bischof

30 Zur Person von Bischof Jaromír (Gebhard) vgl. David KALHOUS, Jaromír-Gebhard, pražský biskup a říšský kancléř (1038-1090): několik poznámek k jeho životu, in: MHB 9 (2003), S. 27-45; Vratislav VANÍĆEK, Vratislav II. (I.): první český král. Praha 2004, bzw. Josef ŽEMLIČKA, Čechy v době knížecí 1034-1198. Praha 2007, S. 89-117.

31 Die Chronik der Böhmen, S. 128 f. (II 42).

32 Vgl. VANÍČEK, Vratislav II., S. 73.

33 „Ubi ante lectum ut vidit Iaromir formaticam semesam et serpilli particulam et cepe super scutellam ac tostam panis buccellam, que forte supera verant presuli post hesternam genticulam, tamquam magna et cruce digna inventa sit culpa, nimis indignans ait: ,Cur vivis parce? Aut cui parcis, o miseret mendice? Me hercle, non decet episcopum vivere parce.“ Die Chronik der Böhmen, S. 121 (II 27).

34 Cosmas bemüht sich nicht, eine Reihe von schwerwiegenden Charakterfehlern seines Bischofs zu verbergen und mitunter erwähnt er sogar respektlos verschiedene gepfefferte Begebenheiten (z. B. das Werfen eines Mitglieds seiner Begleitung in den Rhein bei der Rückreise von der Weihe in Mainz, Die Chronik der Böhmen, S. 118 (II 25). Und wenn er Jaromír als „eingebildet und stolz hochmütig“" charakterisiert (Die Chronik der Böhmen, S. 145 (II 41), müssen wir dies nicht als Beleidigung des Bischofs oder als eine Anmaßung des Prager Propstes verstehen. Zum Selbstbewusstsein der Schreiber in dieser Zeit siehe Marie BLÁHOVÁ, Verfasserbewusstsein in der böhmischen Geschichtsschreibung des Mittelalters, in: Jan A. Aertsen - Andreas Speer (Hgg.), Individuum und Individualität im Mittelalter. Berlin 1996, S. 563-573. 
und zwang Rom, seinen ursprünglichen Standpunkt zu ändern ${ }^{35}$. Die Atmosphäre dieser Veränderungen vermittelt uns Jaromírs Zeitgenosse Cosmas, ebenfalls geformt in den Domschulen und eng verbunden mit dem Leben des Domkapitels. Seine Schilderung von Jaromírs Lebensschicksalen ist also auch ein Zeugnis über das Vordringen des neuen Bischofsideals in die böhmischen Länder, dass hier auch in den folgenden Jahrzehnten resonierte und sich voll in den großen Bischofspersönlichkeiten des 12. Jahrhunderts durchsetzte ${ }^{36}$.

\section{Danksagungen}

Diese Studie ist im Rahmen des Universitätsprogramms PROGRES Q 09: Geschichte der Schlüssel zum Verständnis der globalisierten Welt erschienen.

\section{LITERATURVERZEICHNIS}

\section{Quellen}

Adam von Bremen, Gesta Hammaburgensis ecclesiae pontificum, hg. von Georg Heinrich PERTZ u. a., in: MGH Scriptores (in Folio). Bd. 7: Chronica et gesta aevi Salici. Hannover 1846.

Die Chronik der Böhmen des Cosmas von Prag (Cosmae Pragensis Chronica Boemorum) (MGH Scriptores rerum Germanicarum. Nova series. Bd. 2), hg. von Berthold BRETZHOLZ. Berlin 1923.

Gregor der Große, Regula pastoralis: wie der Seelsorger, der ein untadeliges Leben führt, die ihm anvertrauten Gläubigen belehren und anleiten soll, hg. u. übers. von Georg KUBIS. Graz 1986.

Lampert von Hersfeld, Annales, hg. von Oswald HOLDER-EGGER (MGH Scriptores rerum Germanicarum in usum scholarum separatim editi. Bd. 38). Hannover 1894.

Georg Heinrich PERTZ u. a. (Hg.), Scriptores (in Folio). Bd. 4: Annales, chronica et historiae aevi Carolini et Saxonici. Hannover 1841.

Georg Heinrich PERTZ u. a. (Hg.), Scriptores (in Folio). Bd. 11: Historiae aevi Salici. Hannover 1854.

Thietmari Merseburgensis episcopi Chronicon (MGH Scriptores rerum Germanicarum. Nova series. Bd. 9), hg. von Robert HOLTZMANN. Berlin 1935.

\section{Literatur}

Daniel ALT, Sanctus episcopus: das Bischofsideal von früh- und hochmittelalterlichen Bischofsviten im Spannungsfeld von Anspruch und Wirklichkeit. Herne 2013.

Maria BECKER, Die Kardinaltugenden bei Cicero und Ambrosius: De officiis. Basel 1994.

Walter BERSCHIN, Biographie und Epochenstil im lateinischen Mittelalter. Bd. 1: Von der „Passio Perpetuae“ $z$ u den „Dialogi“ Gregors des Grossen. Stuttgart 1986.

Walter BERSCHIN, Biographie und Epochenstil im lateinischen Mittelalter. Bd. 4: Ottonische Biographie, das hohe Mittelalter: 920-1220 n. Chr. Teil 1: 920-1070. Stuttgart 1999.

Marie BLÁHOVÁ, Verfasserbewusstsein in der böhmischen Geschichtsschreibung des Mittelalters, in: Jan A. Aertsen - Andreas Speer (Hgg.), Individuum und Individualität im Mittelalter. Berlin 1996, S. 563-573.

Lothar BORNSCHEUER, Topik: zur Struktur der gesellschaftlichen Einbildungskraft. Frankfurt am M. 1976.

35 Die Chronik der Böhmen, S. 125 f. (II 30).

${ }^{36} \mathrm{Zu}$ diesem Thema vgl. vor allem Tilman STRUVE, Die Wende des 11. Jahrhunderts: Symptome eines Epochenwandels im Spiegel der Geschichtsschreibung, in: Historisches Jahrbuch 112 (1991), S. 324-365. Weiter auch Aertsen - Speer (Hgg.), Individuum und Individualität im Mittelalter. 
Stephanie COUÉ, Acht Bischofsviten aus der Salierzeit - neu interpretiert, in: Stefan Weinfurter (Hg.), Die Salier und das Reich. Bd. 3: Gesellschaftlicher und ideengeschichtlicher Wandel im Reich der Salier. Sigmaringen 1991, S. 347-414.

Georg DENZLER, Die Kanonikerbewegung und die gregorianische Reform im 11. Jahrhundert, in: Studi gregoriani per la storia della Libertas Ecclesiae 9 (1972), S. 223-237.

Odilo ENGELS, Der Reichsbischof in ottonischer und frühsalischer Zeit, in: Irena Crusius (Hg.), Beiträge $z u$ Geschichte und Struktur der mittelalterlichen Germania Sacra. Göttingen 1989, S. 135-175.

Albrecht FINCK VON FINCKENSTEIN, Bischof und Reich: Untersuchungen zum Integrationsprozess des ottonischfrühsalischen Reiches (919-1056). Sigmaringen 1989.

Josef FLECKENSTEIN, Die Hofkapelle der deutschen Könige. Bd. 2. Stuttgart 1966.

Silke FLORYSZCZAK, Die „Regula pastoralis“ Gregors des Großen: Studien zu Text, kirchenpolitischer Bedeutung und Rezeption in der Karolingerzeit. Tübingen 2005.

Bele FREUDENBERG, Emotionen in einem Werk des 10. Jahrhunderts: die Praeloquia Rathers von Verona, in: Jürgen Sarnowsky (Hg.), Bilder - Wahrnehmungen - Vorstellungen: neue Forschungen zur Historiographie des hohen und späten Mittelalters. Göttingen 2007, S. 17-32.

Stephanie HAARLÄNDER, Vitae episcoporum: eine Quellengattung zwischen Hagiographie und Historiographie, untersucht an Lebensbeschreibungen von Bischöfen des Regnum Teutonicum im Zeitalter der Ottonen und Salier. Stuttgart 2000.

Wolfgang HUG, Elemente der Biographie im Hochmittelalter: Untersuchung zu Darstellungsform und Geschichtsbild der Viten vom Ausgang der Ottonen bis in die Anfänge der Stauferzeit. München 1957.

Reinhold KAISER, Die Gesta episcoporum als Genus der Geschichtsschreibung, in: Anton Scharer Georg Scheibelreiter (Hgg.), Historiographie im frühen Mittelalter. Wien 1994, S. 459-480.

David KALHOUS, Boleslav III.: kníže na konci časů?, in: Tomáš Borovský - Libor Jan - Martin Wihoda (Hgg.), Ad Vitam et Honorem: profesoru Jaroslavu Mezníkovi prátelé a žáci k pětasedmdesátým narozeninám. Brno 2003, S. 221-229.

David KALHOUS, Jaromír-Gebhard, pražský biskup a říšský kancléř (1038-1090): několik poznámek k jeho životu, in: MHB 9 (2003), S. 27-45.

Hatto KALLFELZ, Lebensbeschreibungen einiger Bischöfe des 10.-12. Jahrhunderts. Darmstadt 1973.

Oskar KÖHLER, Das Bild des geistlichen Fürsten in den Viten des 10., 11. und 12. Jahrhundert. Berlin 1935.

Barbara KRZEMIEŃSKA, Krize českého státu na přelomu století, in: ČsČH 18 (1970), S. 497-532.

Johannes LAUDAGE, Priesterbild und Reformpapsttum im 11. Jahrhundert. Köln/Wien 1984.

Helmut LIPPELT, Thietmar von Merseburg: Reichsbischof und Chronist. Köln/Wien 1973.

Friedrich LOTTER, Methodisches zur Gewinnung historischer Erkenntnisse aus hagiographischen Quellen, in: Historische Zeitschrift 229 (1979), S. 298-356.

Marzena MATLA-KOZŁOWSKA, Pierwsi Przemyślidzi i ich państwo (od X do połowy XI wieku): ekspansja terytorialna i jej polityczne uwarunkowania. Poznań 2008.

Dieter von der NAHMER, Die lateinische Heiligenvita: eine Einführung in die lateinische Hagiographie. Darmstadt 2005.

Michel SOT, Gesta episcoporum, gesta abbatum. Turnhout 1981.

Clare STANCLIFFE, St. Martin and his Hagiographer: History and Miracle in Sulpicius Severus. Oxford 1983.

Tilman STRUVE, Die Wende des 11. Jahrhunderts: Symptome eines Epochenwandels im Spiegel der Geschichtsschreibung, in: Historisches Jahrbuch 112 (1991), S. 324-365.

Benedetta VALTORTA, Clavis Scriptorum Latinorum Medii Aevi: Auctores Italiae (700-1000). Firenze 2006.

Vratislav VANÍČEK, Vratislav II. (I.): první český král. Praha 2004.

Stefan WEINFURTER, Neue Forschung zur den Regularkanonikern im deutschen Reich des 11. und 12. Jahrhunderts, in: Historische Zeitschrift 224 (1977), S. 379-397.

Herbert ZIELINSKI, Der Reichsepiskopat in spätottonischer und salischer Zeit (1002-1135). Wiesbaden 1984.

Josef ŽEMLIČKA, Čechy v době knižecí 1034-1198. Praha 2007. 